\title{
Construction of Recombinant Porcine Reproductive and Respiratory Syndrome Virus Expressing CSFV E2 Glycoprotein
}

\author{
Lihua Wang ${ }^{1}$, Rachel Madera ${ }^{1}$, Yulia Burakova ${ }^{1}$, Sterling Buist ${ }^{1}$, Yongming Sang ${ }^{1}$, Jerome Nietfeld ${ }^{2}$, \\ Jamie Henningson ${ }^{2}$, Ada GiselleCino-Ozuna ${ }^{2}$, Wenjie Gong ${ }^{3}$, Changchun $\mathrm{Tu}^{3}$ and Jishu Shi ${ }^{{ }^{*}}$ \\ ${ }^{1}$ Department of Anatomy and Physiology, College of Veterinary Medicine, Kansas State University, Manhattan, KS 66506 USA \\ ${ }^{2}$ Department of Diagnostic Medicine and Pathobiology, College of Veterinary Medicine, Kansas State University, Manhattan, KS 66506 USA
}

${ }^{3}$ Institute of Military Veterinary Medicine, Academy of Military Medical Sciences, Changchun, 130122, China

Received: 06 June, 2018; Accepted: 13 June, 2018; Published: 15 June, 2018

*Corresponding authors: Jishu Shi, Department of Anatomy and Physiology, College of Veterinary Medicine, Kansas State University, 232 Coles Hall, 1620 Denison Ave, Manhattan, KS, 66506. E-mail: jshi@ksu.edu

\begin{abstract}
Classical Swine Fever (CSF) and Porcine Reproductive and Respiratory Syndrome (PRRS) are two highly contagious infectious diseases caused by CSF virus (CSFV) and PRRS virus (PRRSV), respectively. Recombinant PRRSV expressing CSFV E2 glycoprotein could be used for the development of bivalent vaccine, antiviral drug or antibody screening assays against PRRSV and CSFV. In this study, a recombinant PRRSV expressing CSFV E2 glycoprotein (p129-CSFV-E2) was constructed. The E2 gene from CSFV C-strain vaccine was cloned and inserted between ORF1b and ORF2 gene of the PRRSV P129 strain. An additional transcriptional regulatory sequence 6 (TRS6) was inserted following the CSFV E2 for driving the transcription of ORF2. The construct efficiently produced progeny viruses and the expressed CSFV E2 protein was detected by immune staining of infected MARC145 cells. The growth ability of the p129-CSFV-E2 virus is comparable to the parental $\mathrm{p} 129$ virus. The genetic stability and stable expression of CSFV E2 of P129-CSFV-E2 virus could reach 11 passages in cell culture. The results showed that CSFV E2 glycoprotein could be expressed as a separated subgenomic unit in the PRRSV genome. The recombinant P129-CSFV-E2 virus can be useful for the development of novel vaccines, cell-based high throughput antiviral drug and antibody screening system against PRRSV and CSFV.
\end{abstract}

Keywords: CSF; PRRSV; P129; E2 Glycoprotein;

\section{Introduction}

Classical swine fever (CSF) is a highly contagious viral disease of swine, including wild (feral) pigs [1]. E2 glycoprotein is the major antigenic protein of CSFV, which can induce neutralizing antibodies and confer protective immunity in pigs [2]. Our previous studies showed that CSFV E2 from hog cholera lapinized virus C-strain(HCLV, Genotype1.1) can protect pigs from sub genotype heterologous CSFV challenge without the development of fever and growth retardation [3,4].

Porcine reproductive and respiratory syndrome virus (PRRSV) causes respiratory distress with poor growth in piglets and severe reproductive failures in sows [5]. Recently, the development of PRRSV infectious cDNA clones and engineering of the PRRSV genome made possible PRRSV serving as a vaccine vector [6-10]. In this study, we successfully constructed a PRRSV North American (NA) stain P129 based recombinant PRRSV expressing HCLV E2 glycoprotein. Humoral immune responses to PRRSV and CSFV E2 after P129-CSFV-E2 recombinant virus vaccination were tested in pigs.

\section{Material and Methods}

An infectious cDNA clone of pCMV-P129 with two unique restriction sites (Afl II and Mlu I) and a copy of the transcription regulatory sequence of ORF6 (TRS6) between ORFs 1b and 2a was used as the backbone. CSFV E2 gene was amplified from hog cholera lapinized virus C-strain (HCLV, Genotype 1.1) using AccuPrime $^{\mathrm{TM}}$ Pfx Super Mix (Invitrogen, MA, USA) with primers 5'-GAATTGAACTTAAGGCCACCATGGCATTCCTCATCTGCTTGATAA AAGT-3' (the AflII site is underlined) and 5'-CGGAACACGCGTCAA CCAGCGGCGAGTTGTTCTGTTAG-3'(the Mlu I site is underlined). The amplified product was cloned into AflII/Mlu Icut pCMV-P129 to generate plasmid P129-CSFV-E2 (Figure 1A). 80\% confluent MARC-145 cells cultured in 6-well plates were transfected with the plasmid P129-CSFV-E2 using Lipofectamine ${ }^{\mathrm{TM}}$ 2000 (Invitrogen, MA, USA) according to the manufacturer's instructions. After4days of incubation at $37^{\circ} \mathrm{C}$, the cells and supernatants were collected and freeze-thawed for three times and designated passage (P) 1 . The rescued virus was referred to as P129-CSFV-E2 virus.

Virus titer was measured by end-point titration of culture media on MARC- 145 cells. The $50 \%$ tissue culture infectious dose (TCID50) was calculated using the method of Reed and Muench [11].The expression of CSFV E2 was confirmed by indirect immune fluorescence assay (IFA) according to a previous report [3].

Ten conventional Large White-Duroc crossbred weaned specific-pathogen free male piglets (3weeks of age) were 
randomly allotted into 2groups ( $\mathrm{n}=5$ /group). On Day 0 , one group was immunized intramuscularly with a single dose of $5 \times 10^{5}$ TCID $^{50}$ of P129-CSFV-E2 and the other group was mock vaccinated with PBS as control. Pigs were monitored daily for clinical signs (respiratory changes, lethargy, anorexia, and rectal temperature and weight gains). Serum samples were collected every 3 days until 21 days post vaccination (DPV). Animal care and use protocols were approved by Institutional Animal Care and Use Committee (IACUC) at Kansas State University. PRRSVspecific antibody titers were tested by using IDEXX HerdChek ELISA kits as described previously [12]. Measurement of antiCSFV E2 antibody titers in the sera were performed as described previously [3].

\section{Results}

The CSFV E2 gene was inserted between the non-structural and structural genes of the p129 genome using reverse genetic manipulation. The transcription of CSFV E2gene was controlled by TRS2. An additional TRS6 was inserted following the CSFVE2 for driving the transcription of ORF2 (Figure1A). As shown in Figure1B-D, infectious cloneP129-CSFV-E2 efficiently produced progeny viruses with successful expression of CSFV E2 protein in MARC-145 cells. P129-CSFV-E2 virus induced CPE, characterized by cellular rounding and clumping, was visible 24 hours post infection (HPI), and $80-100 \%$ of cells exhibited extensive CPE at 4 DPI tested. Expression of CSFVE2 protein could be detected at 12 (HPI) in some cells by IFA testing. By 4 DPI, almost all the cells were positive for expression of CSFVE2. To examine the growth characters and genetic stability of the recombinant P129CSFV-E2 virus, the P129-CSFV-E2 virus and its parental virus were inoculated into and passaged in MARC- 145 cells. The results showed that the growth ability of the P129-CSFV-E2 virus was comparable to the parental p129 virus (Figure 2A).The genetic stability of CSFV E2 gene insertion was confirmed by RT-PCR and sequencing. Revealing that no mutation was introduced during passages. The stability of CSFVE2 expression was tested by IFA. Indicating the stable expression of CSFVE2through 11 passages in cell culture. The rescued recombinant virus was stable for 11 passages. However, after 11 passages the virus begins losing ability to express HCLV E2 protein when tested by IFA.

From $5 \mathrm{DPV}$ to $12 \mathrm{DPV}$, vaccinated pigs exhibited transient fever (of $40^{\circ} \mathrm{C}-40.7^{\circ} \mathrm{C}$ ). Clinical symptoms diminished after 12 DPV and pigs returned to normal. The PRRSV-associated syndrome, aural cyanosis, commonly known as "blue ear" was not detected at any time point during the experimental period. No clinical signs were observed in any pigs in the control groups. In vaccinated group, PRRSV specific antibodies appeared 1 week after vaccination (Figure 2B). But no CSFV glycoprotein E2 specific antibodies and CSFV neutralizing antibodies appeared during the experiment (data not shown). In control group, PRRSV specific antibodies were not detected during the experiment.
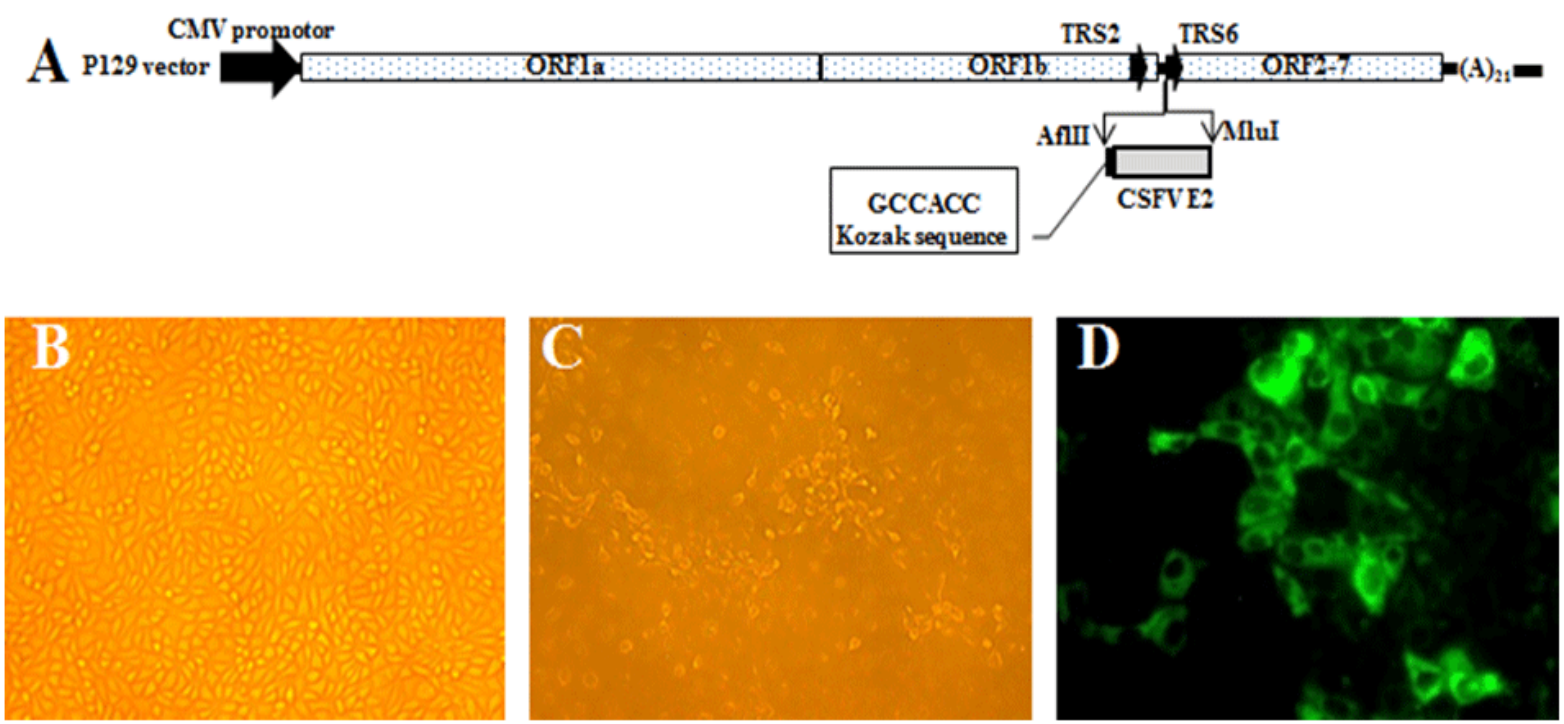

Figure 1: Construction of PRRSV P129 expressing CSFV E2 glycoprotein

(A)Schematic of infectious PRRSV cDNA clone, P129-CSFV-E2.

(B) MARC-145 cell control (40X).

(C) Infection of P129-CSFV-E2 virus (11th passage of the rescued viruses, P11) in MARC-145 cells, 4 DPI (40X).

(D) IFA test the expression of CSFV E2 in P129-CSFV-E2 virus (P11) infected MARC-145 cells; 4 DPI (200X). 


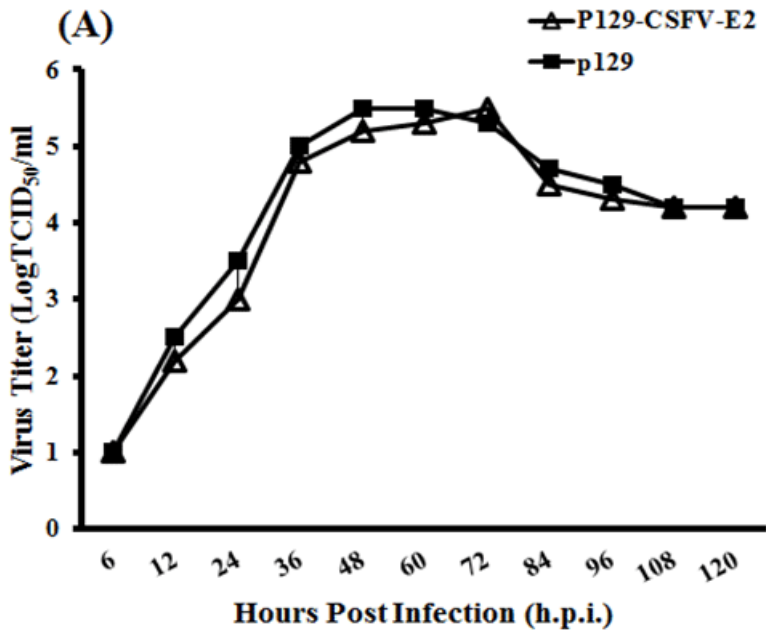

(B)

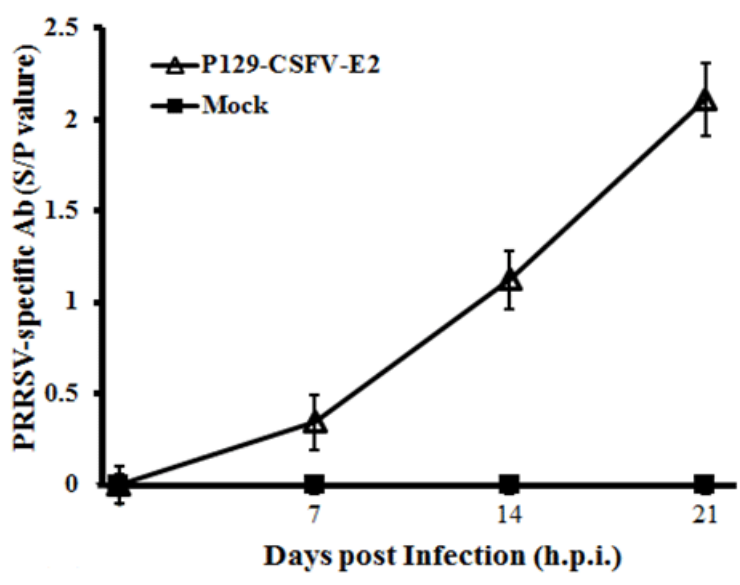

Figure 2: Growth curves of P129-CSFV-E2 recombinant virus and serological response against PRRSV after the recombinant P129-CSFV-E2 virus vaccination.

(A) Multistep growth curves. A multiplicity of infection (MOI) of 0.01 for the parental (p129 virus) and recombinant P129-CSFV-E2 virus (P11) was used to infect fresh MARC-145 cells. Supernatants were harvested at 12, 24, 36, 48, 60, 72, 84, 96, 108, and 120 hpi. Viral titers were determined by the TCID50 and expressed as $\operatorname{LogTCID}_{50} / \mathrm{ml}$.

(B) ELISA (S/P ratio) analysis for humoral immune response against PRRSV specific antibodies of pigs after P129-CSFV-E2 recombinant virus vaccination.

\section{Discussion}

Over the past decade, many efforts have been made to develop PRRSV reverse genetic systems with different PRRSV strains and to construct recombinant viruses using PRRSV as a vector to express foreign genes [6-10, 13-17]. Several reports have highlighted the importance of the insertion position of exogenous genes and insertion size, which could affect genetic stability of foreign gene insertion [7-10, 13-16]. In this study, to obtain a genetically stable recombinant virus that could stably express CSFV E2 glycoprotein, the HCLV E2 gene $(1,086 \mathrm{bp})$ was inserted between ORF1b and ORF2 of P129 genome under the regulation of authentic TRS2, while ORF2 expression was regulated by the extra TRS6 copy. Our results showed that the HCLV E2 gene can be expressed from the extra subgenomic mRNA. The rescued recombinant virus was stable for 11 passages, which confirmed that the region between ORF1b and ORF2 is a suitable site for foreign gene insertion for PRRSV [9, 14]. However, after 11 passages the virus begins losing its ability to express HCLV E2 protein when tested by IFA. Previous reports have indicated that the PRRSV vector has a limited capacity for incorporation of exogenous genes and perhaps the large size of the HCLV E2 gene limits its stability in further passages $[9,14,16]$.

Single-shot intramuscular immunization of P129-CSFV-E2 virus into pigs induced PRRSV-specific antibodies. However, no CSFV E2 specific antibody appeared during the experiment. This phenomenon indicates that the B- and T-cell responses against CSFV E2 glycoprotein were suppressed by P129-CSFV-E2, which is consistent with other reports that PRRSV possesses the capacity to inhibit B- and T-cell responses [18-21]. Although the results indicate that the recombinant P129-CSFV-E2 virus developed in this study may not be a good candidate for bivalent vaccine, it could be used to develop cell-based high throughput antiviral drug and antibody screening system against PRRSV and CSFV, and in studying virus-host interactions.

\section{Acknowledgements}

We thank Dr. Brooke Bloomberg and the rest of the Comparative Medicine staff at Kansas State University for their technical help. Thanks to husbandry staff at the Biosecurity Research Institute at K-State for providing high-level quality of animal care. We thank Dr. Bob Rowland for sharing the PRRSV P129 infectious clone. We thank Dr. Paul Hauer, Dr. Sabrina Swenson, and Ms. Melinda Jenkins-Moore for their assistance in obtaining the CSFV isolates from the National Veterinary Services Laboratories (NVSL), USDA APHIS.

\section{References}

1. Moennig V. Introduction to classical swine fever virus, disease and control policy. Vet Microbiol. 2000;73(2-3):93-102.

2. Van Rijn PA, Bossers A, Wensvoort G, Moormann RJ. Classical swine fever virus (CSFV) envelope glycoprotein E2 containing one structural antigenic unit protects pigs from lethal CSFV challenge. J GenVirol. 1996;77:2737-2745.

3. Madera R, Gong W, Wang L, Burakova Y, Lleellish K, GalliherBeckley A, et al. Pigs immunized with a novel E2 subunit vaccine are protected from sub-genotype heterologous classical swine fever virus challenge. BMC Vet Res. 2016;12:197.

4. Madera RF, Wang L, Gong W, Burakova Y, Buist S, et al. Toward the development of a one-dose classical swine fever subunit vaccine: antigen titration, immunity onset, and duration of immunity. J Vet Sci. 2018;19(3):393-405. 
5. Albina E. Epidemiology of porcine reproductive and respiratory syndrome (PRRS): an overview. Vet Microbiol. 1997;55(1-4):309316.

6. Fang Y, Faaberg KS, Rowland RR, Christopher-Hennings J, Pattnaik $\mathrm{AK}$, Osorio $\mathrm{F}$, et al. Construction of a full-length cDNA infectious clone of a European-like Type 1 PRRSV isolated in the U.S. Adv Exp Med Biol. 2006;581:605-608.

7. Huang Q, Yao Q, Fan H, Xiao S, Si Y, and Chen H. Development of a vaccine vector based on a subgenomic replicon of porcine reproductive and respiratory syndrome virus. J VirolMeth. 2009;160(1-2):22-28.

8. Kim DY, Calvert JG, Chang KO, Horle K, Kerrigan M, Rowland RR. Expression and stability of foreign tags inserted into nsp2 of porcine reproductive and respiratory syndrome virus (PRRSV) Virus Res. 2007;128(1-2):106-114

9. Pei Y, Hodgins DC, Wu J, Welch SK, Calvert JG, Li G, et al. Porcine reproductive and respiratory syndrome virus as a vector: Immunogenicity of green fluorescent protein and porcine circovirus type 2 capsid expressed from dedicated subgenomic RNAs. Virol. 2009;389(1-2):91-99.

10. Yoo D, Welch SK, Lee C, Calvert JG. Infectious cDNA clones of porcine reproductive and respiratory syndrome virus and their potentia as vaccine vectors. Vet. Immunol. Immunopathol. 2004;102(3):143154.

11. Reed LJ, Muench H. A simple method of estimating fifty percent end points. Am. J. Hyg. 1938;27(3):493-497.

12.Li X, Galliher-Beckley A, Nietfeld JC, Faaberg KS, Shi J. MontanideTM Gel01 ST Adjuvant Enhances PRRS Modified Live Vaccine Efficacy by Regulating Porcine Humoral and Cellular Immune Responses. World J Vaccines. 2013;3(1):1-9.

13.Jeeva S, Lee JA, Park SY, Song CS, Choi IS, Lee JB. Development of porcine respiratory and reproductive syndrome virus replicon vector for foot-and-mouth disease vaccine. Clin. Exp. Vaccine Res. 2014;3(1):100-109.
14.Sang YM, Shi JS, Sang WJ, Rowland RR, Blecha F. ReplicationCompetent Recombinant Porcine Reproductive and Respiratory Syndrome (PRRS) Viruses Expressing Indicator Proteins and Antiviral Cytokines. Viruses. 2012;4(1):102-116.

15.Xu YZ, Zhou YJ, Zhang SR, Jiang YF, Tong W, Yu H, et al. Stable expression of foreign gene in nonessential region of nonstructural protein 2 (nsp2) of porcine reproductive and respiratory syndrome virus: applications for marker vaccine design. Vet. Microbiol. 2012;159(1-2):1-10.

16. Yu LX, ZhouYJ, Jiang YF, Tong W, Yang S, Gao F, et al. Construction and in vitro evaluation of a recombinant live attenuated PRRSV expressing GM-CSF. Virol J. 2014;11:201-204.

17. Gao F, Jiang Y, Li G, Zhou Y, Yu L, Li L, et al. Porcine reproductive and respiratory syndrome virus expressing E2 of classical swine fever virus protects pigs from a lethal challenge of highly-pathogenic PRRSV and CSFV. Vaccine. 2018;36(23):3269-3277.

18.Calzada-Nova G, Schnitzlein WM, Husmann RJ, Zuckermann FA. North American porcine reproductive and respiratory viruses inhibit type I interferon production by plasmacytoid dendritic cells. J Virol. 2010;85(6):2703-2713.

19. Costers S, Lefebvre DJ, Goddeeris B, Delputte PL, Nauwynck HJ. Functional impairment of PRRSV-specific peripheral CD3+CD8 high cells. Vet Res. 2009;40(6):40-46.

20.Darwich L, Díaz I, Mateu E. Certainties, doubts and hypotheses in porcine reproductive and respiratory syndrome virus immunobiology. Virus Res. 2010;154(1-2) :123-132

21. Kimman TG, Cornelissen LA, Moormann RJ, Rebel JM, StockhofeZurwieden N. Challenges for porcine reproductive and respiratory syndrome virus (PRRSV) vaccinology. Vaccine. 2009;27(28):37043718. 